\title{
Experimental characterization of a quasi-coherent turbulent structure in the edge plasma in Wendelstein 7-X
}

\author{
X. Han ${ }^{1,2}$, A. Krämer-Flecken ${ }^{1}$, T. Windisch ${ }^{3}$, M. Hirsch ${ }^{3}$, G. \\ Fuchert $^{3}$, J. Geiger ${ }^{3}$, O. Grulke ${ }^{3}$, S. Liu ${ }^{1,2}$, K. Rahbarnia ${ }^{3}$, and \\ the W7-X team $^{3} \ddagger$ \\ ${ }^{1}$ Forschungszentrum Jülich GmbH, Institut für Energie- und Klimaforschung \\ Plasmaphysik, Partner of the Trilateral Euregio Cluster (TEC), 52425 Jülich, \\ Germany \\ ${ }^{2}$ Institute of Plasma Physics, Chinese Academy of Sciences, 230031 Hefei, Anhui, \\ People's Republic of China \\ ${ }^{3}$ Max-Planck-Institut für Plasmaphysik, 17491 Greifswald, Germany \\ E-mail: xi.han@fz-juelich.de
}

\begin{abstract}
A quasi-coherent mode (QCM) near the plasma edge is characterized experimentally by measuring the density fluctuation via a poloidal correlation reflectometer (PCR) in Wendelstein 7-X. This QCM frequency descends from $25 \mathrm{kHz}$ near the separatrix to $10 \mathrm{kHz}$ as the cutoff position penetrates toward the core. A similar mode structure is also observed in the spectra of ECE edge channels and Mirnov coil signals, which shows a clear correlation with respect to the PCR measurement. The mode evaluation yields a range for the poloidal mode number of $8 \leq \mathrm{m} \leq 25$, and for the normalized mode scale $\mathrm{k}_{\perp} \rho_{\mathrm{s}} \leq 0.1$, which suggest a drift type instability. The QCM structure appears in the standard and narrow-mirror configurations (both with the rotational transform $\iota=5 / 5$ in the boundary), but is absent in the high- $\iota$ configuration (boundary $\iota=5 / 4$ ). The impact of plasma parameters on the QCM is discussed. It is observed that the QCM frequency and amplitude can be affected by the edge magnetic topology and plasma parameters. The $\mathrm{f}_{\text {mode }}$ shifts upward during the negative and non-stellarator-symmetric control coil current scans, which may be attributed to the effect of the $\mathrm{E} \times \mathrm{B}$ shear, $\mathrm{E}_{\mathrm{r}}$, and $\mathrm{n}_{\mathrm{e}}$ in the plasma edge region.
\end{abstract}

Keywords: quasi-coherent mode, poloidal correlation reflectometer, W7-X

Submitted to: Nucl. Fusion

$\ddagger$ Members of the W7-X team are listed in R. C. Wolf et al [1]. 


\section{Introduction}

Turbulence has been proved to play a significant role in the edge cross-field heat and particle transport in magnetic confinement devices[2]. The turbulence can be driven by, for instance, density gradients[3], energetic particles[4], or plasma current[5]. Characterization of the turbulence regime is advantageous for a better understanding of its impact on the overall energy confinement[6]. The socalled 'quasi-coherent mode' (QCM) is identified as a distinct peak appearing in the temperature, density, and/or magnetic fluctuation spectrum. In general, the QCM central frequency covers a bandwidth from tens to hundreds of $\mathrm{kHz}$, and has been observed in a wide range of core and edge regions in many tokamaks[7, 8, 9] and stellarators[10, 11, 12, 13]. For instance, the electromagnetic mode at $\mathrm{f}_{\text {mode }} \approx 26.5 \mathrm{kHz}$ observed in the pedestal region of EAST tokamak is responsible for an outward particle transport in the low wavenumber range $\left(\mathrm{k}_{\perp}<3 \mathrm{~cm}^{-1}\right)$ [14]. Also in the TJ-II stellarator a quasi-coherent electrostatic mode at $\mathrm{f}=20 \sim 120 \mathrm{kHz}$ was observed to contribute to a small fraction of the turbulent particle flux for $\mathrm{k}_{\theta}<$ $3 \mathrm{~cm}^{-1}[15]$. From the modeling point of view, the coupling between ion drift waves and electron drift-acoustic waves implies an important destabilization effect for a driftballooning mode, which might be related to the quasi-coherent edge instabilities at the H-mode pedestal[16]. In Wendelstein 7-X (W7-X) stellarator, a three-dimensional geometry of magnetic field is designed for a quasi-isodynamic equilibrium at high plasma beta. The magnetic configuration has been confirmed to control bootstrap currents and collisional transport by mean of changes in the magnetic mirror ratio in the first experimental campaign of
W7-X[17]. The neoclassical transport induced by the bounce-averaged radial drift of trapped particles is strongly reduced. The magnetic field strength and the local curvature $\kappa$ are shifted with respect to each other, and the turbulent structure can be distinguished between the ion-temperature gradient (ITG) and trapped-electron mode (TEM) by examining the scale length of the turbulence[18].

In the first W7-X campaign with the limiter configuration, rotations and radial electric fields are evaluated and compared with the neoclassical calculations by measuring the poloidal propagation of density fluctuations[19]. An electromagnetic coherent mode at $\mathrm{f} \approx 7 \mathrm{kHz}$ in the plasma edge is analysed by means of both the poloidal correlation reflectometer (PCR) and the Langmuir probes[20]. The CAS3D simulation suggests that the coherent fluctuation is attributed to the stable MHD mode and Alfvén wave[21]. The existence of the mode relies on the heating power, indicating a dependency of the electron temperature and pressure profiles.

In the island-divertor configurations, turbulence transport in the scrape-off layer has been proved to be influenced by the magnetic topology[22]. A broadband turbulence in a frequency range of 40 to $120 \mathrm{kHz}$ is observed near the island centre. In the edge density gradient region, a QCM structure at $\mathrm{f}_{\text {mode }}=10$ to 25 $\mathrm{kHz}$ is also observed recently. In this paper, the mode behavior is investigated by analysing the cross-correlation spectrum via the density fluctuation measurement of PCR. Results indicate that the QCM may be ascribed to a drift type instability, and the mode frequency can be modulated by the edge magnetic topology. The remaining of this paper is outlined here: a brief introduction to the experimental setup is in section 2. The experimental observation of the QCM structure is presented in section 
3. Then the mode behavior is characterised in section 4. The upward shifting of the QCM frequency is described in section 5 followed by a summary in section 6 .

\section{Experimental setup on $\mathrm{W} 7-\mathrm{X}$}

The PCR diagnostic has been installed on W7-X for the purpose of investigations on turbulence behavior at the interface between core and edge plasmas[23]. The transmitted wave covers a frequency range from 22 to 40 $\mathrm{GHz}$ in an ordinary polarization (O-mode), which corresponds to a cut-off density range of $\mathrm{n}_{\mathrm{e}, \text { cutoff }}=0.6 \sim 2 \times 10^{19} \mathrm{~m}^{-3}$. The output waveform is controlled remotely to realize an either constant or hopped frequency scan.

The front-end of PCR is installed in the highly elongated bean-shaped cross section in W7-X. The PCR's antenna array consists of five pyramidal horns, which are integrated onto a $1.5 \mathrm{~m}$ long plug-in module. Figure 1 shows a Poincaré plot of magnetic field lines at the cross-section of the PCR in the standard reference configuration (so-called EJM), where all modular coil currents are the same, and the boundary rotational transform is $\iota=1$ with a $5 / 5$ island chain. The launcher horn (labeled as $\mathrm{A}$ ) is positioned at $R=6.5 \mathrm{~m}, z=-0.157$ $\mathrm{m}$, and the other four receiver horns (labeled as B, C, D and E) surrounds the launcher. All the antennas have a joint focus point with respect to the sight line of antenna $\mathrm{A}$ at $(\mathrm{R}, \phi, \mathrm{z})=$ $\left(6.0 \mathrm{~m}, 71.05^{\circ},-0.104 \mathrm{~m}\right)$. A broad radiation pattern of the antennas has been included in their design in order to compromise the slight toroidal and poloidal shifts for the reflection points. Therefore the four receiver horns can collect the reflected wave with a poloidal discrimination at the corresponding cutoff layers. The radial-temporal characteristics of the rotation velocity and the radial electric field $\left(E_{r}\right)$ can be achieved based on this poloidal array of receivers[24]. The PCR system has been operated successfully and routinely since the first W7-X campaign with the limiter configuration. The turbulence activities in the scrape-off layer (SOL) and plasma core have been studied[25]. The perpendicular velocity $\left(\mathrm{v}_{\perp}\right)$ and radial electron field $\left(\mathrm{E}_{\mathrm{r}}\right)$ profiles in the vicinity of magnetic islands have been measured successfully via PCR [26] and compared well with the combined probe[27].

The PCR measurement is related to the cutoff density, which can be calculated. To obtain the radial position of the cutoff density, a density profile is needed. In W7-X the density profile is measured by the Thomson Scattering (TS) diagnostic[28]. Concerning the toroidal angle between PCR and TS, the $n_{e}$ profile needs to be mapped to the effective minor radius $\left(\mathrm{r}_{\mathrm{eff}}\right)$ coordinate. Here the $r_{\text {eff }}$ is defined as $r_{\text {eff }}=a_{\text {LCFS }} \sqrt{s}$, with the minor radius of the LCFS ( $\mathrm{a}_{\mathrm{LCFS}}$ ), and the normalized toroidal flux s, which is normalized to the toroidal flux enclosed by the plasma boundary $\Phi_{\text {LCFS }}$. The magnetic surfaces as well as the LCFS are calculated using the VMEC code[29] whose major assumption is that the equilibrium region is described by nested closed flux surfaces, i.e. there are no islands or stochastic regions. If islands of a main resonance are used to form a separatrix limiting the plasma by intersecting the islands with divertor structures, the VMEC-calculation has to be restricted to the good flux surface region. Mapping of diagnostic information outside the VMECdomain is in this case not possible, first because there are no VMEC-flux surfaces and second because the island structure and the SOL-plasma can no longer be described so simple as the core plasma. In our case we take 
the local TS measurements outside the LCFS, and extend the $\mathrm{r}_{\text {eff }}$ by adding the differential of the two adjacent TS volume in the openfield line region to the $r_{\text {eff }}$ coordinate. This implies an uncertainty for the $\mathrm{r}_{\text {eff }}$ estimation outside the LCFS. However, this uncertainty is accepted for a better intuitive presentation of profiles. The TS density profile shape is approached using an exponential function:

$\mathrm{n}_{\mathrm{e}}\left(\mathrm{r}_{\mathrm{eff}}\right)=\mathrm{n}_{\mathrm{e} 0} \exp \left(-\mathrm{a}_{1} \mathrm{r}_{\text {eff }}^{2}-\mathrm{a}_{2} \mathrm{r}_{\text {eff }}^{4}-\mathrm{a}_{3} \mathrm{r}_{\text {eff }}^{6}\right)$

The $n_{e}$ profile is averaged within each of the PCR sweeping cycles. The TS $n_{e}$ values are cross-calibrated with the interferometer measurements[30].

The magnetic equilibrium is obtained by taking planar and non-planar coil currents to find the reference equilibrium. The VMEC configuration with the most similar coil current setting can be selected in the database. The locations of the LCFS and the island are sensitive with respect to the edge magnetic topology, which can be adjusted via, e.g. the main coil current, the control coil currents, and the plasma $\beta$. Nevertheless, the shear layer region can be inferred from the change of the sign in the $\mathrm{E}_{\mathrm{r}}$ profile, and the QCM is visible inside the LCFS.

\section{QCM observation}

In W7-X OP1.2 campaign with the islanddivertor configuration, a QCM structure at the frequency range of 10 to $25 \mathrm{kHz}$ is frequently observed via several diagnostics. Figure 2 shows a discharge with the QCM observation in the EJM configuration, where the ECRH heating power $\mathrm{P}_{\mathrm{ECRH}}=3 \mathrm{MW}$, and the lineintegrated density $\int \mathrm{n}_{\mathrm{e}} \mathrm{dl} \approx 3 \times 10^{19} \mathrm{~m}^{-2}$. In figure $2(\mathrm{c})$ of the cross-power spectrum using receiver E-C combination, the QCM appears repeatedly when the transmitted frequency exceeds $36 \mathrm{GHz}$ in each scan, which corresponds a cutoff density of $1.6 \times 10^{19} \mathrm{~m}^{-3}$. The mode frequency initiates at $\mathrm{f} \approx 25 \mathrm{kHz}$, and gradually decreases to $10 \mathrm{kHz}$ as the cutoff position moves toward the core. The QCM bandwidth is $\delta \mathrm{f} / \mathrm{f} \approx 0.25$.

Figure 2(b) is the time trace of turbulence delay time $(\tau)$ between $\mathrm{E}$ and $\mathrm{C}$ receivers. Here, $\tau$ is related to the time at which a turbulent structure propagates along the distance between the reflection points of two receivers on the cut-off surface[31]. It can be calculated via the peaking point in the cross-correlation function (CCF) $\tau=$ $\arg \left(\max _{\delta \mathrm{t}}\left(\left|\mathrm{E}_{1} \star \mathrm{E}_{2}\right|\right)(\delta \mathrm{t})\right) .\left|\mathrm{E}_{1}\right|$ and $\left|\mathrm{E}_{2}\right|$ denote the time series of fluctuations from the two receivers. In each individual scan, as the transmitted wave penetrates toward the core, the change of sign in $\tau$ implies a transmitted wave passes through the shear layer by assuming that the direction of the turbulent flow is opposite in between. Therefore the shear layer region can be identified near the corresponding positions where $\tau=0 \mu \mathrm{s}$.

This QCM structure can be seen in the cross-power spectrum of each two receiver combinations of PCR. The mode amplitude becomes strong in the density gradient region inside the LCFS. A magnetic fluctuation at the frequency range of $\mathrm{f} \approx 10 \sim 25 \mathrm{kHz}$ can be seen in the auto-power spectrum of the Mirnov coil signal in figure $3(\mathrm{a})$, which is in the same frequency range of the QCM. Due to a missing radial localization of the measurement of the magnetic fluctuation in the Mirnov coil signal, it is impossible to see the mode frequency shifting along the radial position. The crosscorrelation coefficient is calculated by taking the signals of the $\mathrm{E}$ receiver and the Mirnov coil. A clear correlation coefficient at $\mathrm{f} \approx$ $10 \mathrm{kHz}$ is obtained as is shown in the figure $3(\mathrm{c})$, which shows an evidence that the QCM is an electromagnetic mode. 
A similar mode structure is also observed in the radiation temperature $\left(\mathrm{T}_{\mathrm{rad}}\right)$ fluctuation spectra among the edge channels of the electron cyclotron emission (ECE) diagnostic. Figure 4 (a) shows a $\mathrm{T}_{\text {rad }}$ spectrum at the high-field side (HFS) plasma edge, where the ECE emission frequency is $155 \mathrm{GHz}$. The mode appears from $\mathrm{t}=0.2 \mathrm{~s}$ and keeps at $\mathrm{f} \approx 15 \mathrm{kHz}$ constantly. By calculating the $\mathrm{T}_{\mathrm{rad}}$ spectra of ECE in the same time window where the QCM is observed in the PCR, the mode frequency is compared among ECE signals. The ECE frequency is inversely proportional to the major radius $\left(f_{\mathrm{ECE}} \propto\right.$ $1 / \mathrm{R})$, the corresponding cutoff position moves toward core as the ECE frequency decreases from the HFS. In figure 4(b), the ECE signal at $\mathrm{f}_{\mathrm{ECE}}=155 \mathrm{GHz}$ is located at several centimeters inside LCFS. It is observed that a radial distribution of the mode from the edge to the half radius, and the mode frequency descends along with the $\mathrm{f}_{\mathrm{ECE}}$ moves from the edge. In figure 4(c) of the cross-correlation coefficient between receiver $\mathrm{B}$ and ECE signal, a weak peak at $\mathrm{f} \approx 14 \mathrm{kHz}$ can be seen, which indicates that the mode in ECE spectrum could be the same one that we observed in the PCR signal.

\section{Characterization of the QCM}

Figure 5 shows the cross-phase and wavenumber calculations using the receiver E-C combination at the $\mathrm{n}_{\mathrm{e}, \text { cutoff }} \approx 1.6 \times 10^{19} \mathrm{~m}^{-3}$. The corresponding cutoff position is $\mathrm{r}_{\text {eff }} \approx 0.51 \mathrm{~m}$, and the poloidal separation of the E-C antenna is $\delta \mathrm{s}=1.15 \mathrm{~cm}$. As shown in figure $5(\mathrm{a})$, the mode central frequency is at $\mathrm{f}_{\text {mode }} \approx 23 \mathrm{kHz}$ with a bandwidth of $\Delta \mathrm{f}=6 \mathrm{kHz}$ and the $\Delta \mathrm{f} / \mathrm{f} \approx 0.28$. In the figure $5(\mathrm{~b})$, the cross phase at $-50 \mathrm{kHz}<\mathrm{f}<50 \mathrm{kHz}$ yields a negative slope. By linear fitting the cross-phase, the de- lay time can be evaluated from the slope of the fitted cross-phase, which is $\tau=(\mathrm{d} \phi / \mathrm{df}) / 2 \pi \approx$ $-1.92 \sim-2.09 \mu \mathrm{s}$. This value is consistent with the $\tau$-value obtained from the CCF calculation in figure 2(b). The wavenumber spectral density $\left(\mathrm{S}\left(\mathrm{k}_{\theta}, \mathrm{f}\right)\right)$ can be calculated via a two-point cross-correlation technique[32]. It is found in figure $5(\mathrm{c})$ that the poloidal wavenumber of the $\mathrm{QCM}$ is $\mathrm{k}_{\theta} \approx-0.22 \mathrm{~cm}^{-1}$ at $\mathrm{f}_{\text {mode }}=$ $23 \mathrm{kHz}$. The negative $\mathrm{k}_{\theta}$ value reveals an electron diamagnetic drift direction of QCM in the laboratory frame. The perpendicular velocity is deduced to be $\mathrm{v}_{\perp}=\omega / \mathrm{k} \approx 6.5 \mathrm{~km} / \mathrm{s}$. Here, the $\mathrm{v}_{\perp}$ is the perpendicular velocity of the turbulence, which is a contribution from both the $\mathrm{E} \times \mathrm{B}$ velocity $\left(\mathrm{v}_{\mathrm{E} \times \mathrm{B}}\right)$ and the phase velocity ( $\left.v_{\text {phase }}\right)$ of the turbulence. Experimentally in the delay time estimation, $\mathrm{v}_{\perp}$ can be measured via $\mathrm{v}_{\perp}=\delta \mathrm{s} / \tau_{\mathrm{m}}$, where the $\delta$ s denotes the distance of the two receivers at the corresponding cutoff layer, and the $\tau_{\mathrm{m}}$ is the maximum delay time in the CCF. In order to calculate the $\delta \mathrm{s}$, one needs the knowledge of the magnetic field pitch angle $\alpha$, i.e., the tilting angle of the magnetic field line, which is defined to be $\alpha=\arctan \left(\mathrm{B}_{\mathrm{p}} / \mathrm{B}_{\mathrm{t}}\right)$, the ratio of the polodial magnetic filed $\left(\mathrm{B}_{\mathrm{p}}\right)$ and the toroidal one $\left(B_{t}\right)$. It can be obtained via tracing the microwave ray and the geometric simulations[33]. We learn from figure $5(\mathrm{~b})$ that $\mathrm{v}_{\text {phase }}$ can be neglected in the range of $-50 \mathrm{kHz} \leq \mathrm{f} \leq 50 \mathrm{kHz}$, therefore we assume $\mathrm{v}_{\perp}=\mathrm{v}_{\mathrm{E} \times \mathrm{B}}$ in the following analysis.

The QCM behaviors are further characterized by calculating its poloidal mode number $(\mathrm{m})$, the normalized gyroradius $\left(\mathrm{k}_{\perp} \rho_{\mathrm{i}}\right)$, and the effective correlation length $\left(l_{\text {eff }}\right)$, results are depicted in the figure 6 . The poloidal mode number is calculated to be $\mathrm{m}=\mathrm{k}_{\perp} \mathrm{s} / 2 \pi$ with the $\mathrm{s}$ the circumference at the corresponding cutoff position of the PCR. By extracting the QCM frequency from the figure $2(\mathrm{c})$, the poloidal 
mode number for the QCM is obtained to be $8 \leq \mathrm{m} \leq 25$.

The electron temperature at plasma edge is $\mathrm{T}_{\mathrm{e}} \approx 30 \pm 10 \mathrm{eV}$ in the TS measurement. The normalized gyroradius is then estimated to be $\rho_{\mathrm{s}}=2 \pi \sqrt{\mathrm{T}_{\mathrm{e}} \mathrm{m}_{\mathrm{i}}} / \mathrm{eB} \approx 0.23 \mathrm{~cm}$ by using the edge toroidal magnetic field from VMEC and assuming the $T_{i}=T_{e}$. Thus, the normalized mode scale is derived to be $\mathrm{k}_{\perp} \rho_{\mathrm{i}} \leq 0.1$, which is shown in the figure $6(\mathrm{~b})$. According to the drift-wave turbulence theory, the frequency for the drift wave is in the range of $\omega \sim$ $\mathrm{k}_{\perp} \mathrm{v}_{\text {drift }}$, and the turbulent size scale is in a range of $\mathrm{k}_{\perp} \rho_{\mathrm{s}}<0.1[34,35]$. Here $\mathrm{k}_{\perp}$ is the perpendicular wavenumber of the turbulence, $\mathrm{v}_{\text {drift }}$ is the diamagnetic drift velocity, and the $\rho_{\mathrm{s}}$ is the drift-wave gyroradius parameter. The result shows that the experimental estimation of $\mathrm{k}_{\perp} \rho_{\mathrm{i}}$ is quantitatively consistent with the drift-wave size scale. The effective correlation length is defined to be $l_{\text {eff }}=\left|\mathrm{v}_{\perp} \tau_{\mathrm{a}}\right|$ with $\tau_{\mathrm{a}}$ the auto-correlation time. In W7-X, the $\tau_{\mathrm{a}}$ can be measured via the auto-correlation function of the PCR receiver in the laboratory frame. Experimentally, we take the average $\tau_{\mathrm{a}}$ value from all the four receivers, and assume its standard deviation as the errorbar. The result yields a $\tau_{\mathrm{a}}$ in the range of $\tau_{\mathrm{a}} \approx 4.7 \sim 6.9 \mu \mathrm{s}$ near the separatrix. Thus the $l_{\text {eff }}$ spans from $2.1 \mathrm{~cm}$ to $4.2 \mathrm{~cm}$, as is shown in the figure $6(\mathrm{c})$. A linear distribution of the $\mathrm{m}, \mathrm{k}_{\perp} \rho_{\mathrm{i}}$, and $\mathrm{l}_{\mathrm{eff}}$ is mainly due to the QCM frequency shifting radially, which can be seen from the mode radial structure in the figure $6(\mathrm{~d})$. The radial size of QCM is $\Delta \mathrm{r}_{\text {eff }} \sim 3.5 \mathrm{~cm}$, which covers the density gradient region in the plasma edge.

In the W7-X operation phase with the island-divertor configuration, the appearance of QCM prefers a certain parameter range. As an example, the figure 7 compares the cross-power spectrum using the receiver E-C combination to show how the QCM structure evolves with respect to the $\mathrm{P}_{\mathrm{ECRH}}$ and the toroidal plasma current $\mathrm{I}_{\text {tot }}$. In the discharge \#180731008 with a constant $\mathrm{P}_{\mathrm{ECRH}}$, the QCM can be seen during the increase of $\mathrm{I}_{\text {tot }}$. However, in the discharge\#180801021, the $\mathrm{P}_{\mathrm{ECRH}}$ changes from $2 \mathrm{MW}$ up to $4 \mathrm{MW}$, while the $\mathrm{I}_{\text {tot }}$ keeps increasing and slowly saturating. A clear QCM structure appears in the first ECRH step, its amplitude becomes weaker gradually in the second power step. After $5 \mathrm{~s}$, the QCM becomes invisible, where both $\mathrm{P}_{\mathrm{ECRH}}$ and $\mathrm{I}_{\text {tot }}$ are high.

Moreover, this QCM is only observed in the EJM and the narrow-mirror (KKM) configurations, both of which have a boundary iota $\iota=5 / 5$, there is no QCM observed in the high-iota configuration (FTM, boundary $\iota=$ $5 / 4$ ). In W7-X, the magnetic configurations are achieved by adjusting the coil current setting of the non-planar coils, planar coils and trim coils. The magnetic topology has an influence on the turbulence transport in the SOL region, which has been measured by the combined probe in W7-X[22]. Our observation indicates that the magnetic topology as well as the plasma parameter can influence the turbulence structure near the separatrix.

\section{5. effect of control coil currents on QCM}

The edge magnetic island can be adjusted via a set of control coil currents $\left(\mathrm{I}_{\mathrm{cc}}\right)$ in W7-X. This set consists of ten stellarator symmetric control coils, two in each period, which can either enlarge the magnetic island size by applying both positive currents, or reduce the island size with both negative currents[36]. The non-stellarator-symmetric $I_{\mathrm{cc}}$ setup, i.e. with inverted currents in two neighboring half modules, is capable to sweep the island position poloidally, i.e. the island phase. The 
effect of $I_{c c}$ has been shown to influence the heat load on the divertor targets[37]. Here we observe that an $\mathrm{I}_{\mathrm{cc}}$ scan can modulate the QCM frequency too. In the figure 8, the mode frequency evolutions in three different $\mathrm{I}_{\mathrm{cc}}$ setups are compared. All these three discharges have similar plasma parameters. in the discharge \#11 (figure 8(c)) after $5.5 \mathrm{~s}$, $\mathrm{I}_{\mathrm{cc}}$ starts to ramp up to $1.4 \mathrm{kA}$, while the QCM frequency keeps constant at $\mathrm{f}_{\text {mode }} \approx$ $18 \mathrm{kHz}$. However, in the discharge \#12, where $\mathrm{I}_{\mathrm{cc}}$ is set to ramp negatively with an $\mathrm{I}_{\mathrm{cc}}$ ramping rate $\left|\mathrm{dI}_{\mathrm{cc}} / \mathrm{dt}\right| \approx 155 \mathrm{~A} / \mathrm{s}$, the $\mathrm{QCM}$ frequency ascends from $18 \mathrm{kHz}$ to $24 \mathrm{kHz}$. In the discharge \#13 with the non-stellaratorsymmetric $\mathrm{I}_{\mathrm{cc}}$ scan, the QCM frequency also raises to $22 \mathrm{kHz}$.

During the $\mathrm{I}_{\mathrm{cc}}$ scans, we observe that the QCM frequency shift is accompanied by a fluctuation modulation in the frequency range of $\mathrm{f}<10 \mathrm{kHz}$, as can be seen in the figure 8 (d) and (e). The turbulence decorrelation time $\left(\tau_{\mathrm{dc}}\right)$ is defined, within the laboratory frame of reference, as the time where the envelope of all the CCF combinations has reduced to the $1 / \mathrm{e}-$ level[33]. It is estimated to be $\tau_{\mathrm{dc}} \approx 4.5 \mu \mathrm{s}$ at the QCM location, whose inverse absolute value is comparable with respect to the $\mathrm{E} \times$ B flow shearing rate $\omega_{\mathrm{E} \times \mathrm{B}}=\left|\partial \mathrm{V}_{\perp} / \partial \mathrm{r}_{\text {eff }}\right| \approx$ $210 \times 10^{3} \mathrm{~s}^{-1}$ at the same location. This low frequency turbulence modulation may be attributed to the $\mathrm{E} \times \mathrm{B}$ flow shear effects[38]. It is found that the $\mathrm{E} \times \mathrm{B}$ shear can also alter the turbulence drive and spreading in the edge region, which has been measured quantitatively in the TJ-II stellarator [39]. Unfortunately it is not possible to investigate the QCM's radial propagation based on the present PCR measurement, since the radial velocity fluctuation is required to estimate the turbulence drive and spreading rates. This issue will be addressed in the future W7-
$\mathrm{X}$ campaigns since then a radial correlation reflectometer will be developed for W7-X.

Figure 9 (a) and (b) show the comparison of the $n_{e}$ and $E_{r}$ profiles in the three different $I_{c c}$ scans. Before the $I_{c c}$ ramps, both $n_{e}$ and $E_{r}$ profiles are in the similar level for all the three discharges, which are depicted by the black triangles. Taking one individual PCR scan in the time window of 13.6 to $14.3 \mathrm{~s}$ where the $\mathrm{I}_{\mathrm{cc}}$ ramps up to $1.2 \mathrm{kA}$ non-stellarator-symmetric (\#11 and \#12) or symmetric (\#13), the $n_{e}$ and $E_{r}$ profiles evolve differently. The edge $n_{e}$ remains unchanged in the case of the positive $\mathrm{I}_{\mathrm{cc}} \mathrm{scan}(\# 11)$, and the $\mathrm{E}_{\mathrm{r}}$ profile stays steadily. In the negative $\mathrm{I}_{\mathrm{cc}}$ scan $(\# 12)$, the two of the edge TS measurements between $\mathrm{r}_{\text {eff }}=0.45 \mathrm{~m}$ and $0.51 \mathrm{~m}$ increase, which leads to an outward moving of the PCR's cutoff position. It can be seen in the $E_{r}$ profile (the red curve in figure $9(\mathrm{~b})$ ), and the $\mathrm{E}_{\mathrm{r}}$ shear becomes stronger in the shear region. In the symmetrical $\mathrm{I}_{\mathrm{cc}}$ scan (\#13), the edge $n_{e}$ measurements also increase inside the LCFS, it is comparable to the one in the negative $\mathrm{I}_{\mathrm{cc}}$ case. Meanwhile, the $\mathrm{E}_{\mathrm{r}}$ shear becomes weak, and the shear region moves outward by $\sim 1.5 \mathrm{~cm}$.

\section{Summary}

To summarize our work, a QCM structure near the plasma edge has been studied experimentally in W7-X, which behaves as a drift wave with its frequency decreasing from $25 \mathrm{kHz}$ near the separatrix to $10 \mathrm{kHz}$ toward the core. The QCM structure shows a preference on the magnetic configuration, implying a dependence of the edge magnetic topology effect. Furthermore, it is observed that in the cases of symmetrical and negative non-stellarator-symmetric $\mathrm{I}_{\mathrm{cc}}$ scans, the QCM frequency ascends along with the increase of the coil current. This might be attributed 
to the effects of the $E_{r}$ shear in the shear region. Nevertheless, this observation points towards that the turbulence structure could be dominated by the topology effects, which will directly influence the turbulence transport at plasma edge region. The observation in this paper may shed the light, from the experimental point of view, on the turbulence transport near the separatrix in the 3D magnetic field line geometry, and will be investigated further in the future W7-X campaigns.

\section{Acknowledgments}

This work has been carried out within the framework of the EUROfusion Consortium and has received funding from the Euratom research and training programme 2014-2018 and 2019-2020 under grant agreement No 633053. The views and opinions expressed herein do not necessarily reflect those of the European Commission. And was supported by the National Natural Science Foundation of China under Grant No. 11605235.

\section{Reference}

[1] Wolf R et al. 2017 Nuclear Fusion 57102020 URL http://stacks.iop.org/0029-5515/ $57 / i=10 / a=102020$

[2] Kardaun O, Thomsen $\mathrm{K}$ and Chudnovskiy A 2008 Nuclear Fusion 48099801 URL https : //doi.org/10.1088\%2F0029-5515\% 2F48\%2F9\%2F099801

[3] Tynan G R, Fujisawa A and McKee G 2009 Plasma Phys. Control. Fusion 51 ISSN 07413335 URL https: //iopscience.iop.org/article/10.1088/ 0741-3335/51/11/113001/pdf

[4] Breizman B N and Sharapov S E 2011 Plasma Phys. Control. Fusion 53 ISSN 07413335

[5] Leonard A W 2014 Phys. Plasmas 090501 1-24 ISSN 15361055 URL http://dx.doi.org/10. $1063 / 1.4894742$
[6] Hidalgo C 2011 Plasma Physics and Controlled Fusion 53074003 URL http://stacks.iop. org $/ 0741-3335 / 53 / i=7 / a=074003$

[7] Han X et al. 2014 Physics of Plasmas 21 102504 (Preprint https://doi.org/10.1063/ 1.4897923) URL https://doi.org/10.1063/ 1.4897923

[8] Zhong W L et al. 2016 Physics of Plasmas 23 060702 (Preprint https://doi.org/10.1063/ 1.4953427) URL https://doi.org/10.1063/ 1.4953427

[9] Arnichand $\mathrm{H}$ et al. 2016 Plasma Physics and Controlled Fusion $\mathbf{5 8} 014037$ URL http: //stacks . iop.org $/ 0741-3335 / 58 / i=1 / a=014037$

[10] Dewhurst J M et al. 2008 Plasma Physics and Controlled Fusion 50095013 URL http://stacks.iop.org/0741-3335/50/i=9/ $\mathrm{a}=095013$

[11] Hidalgo C et al. 2002 New Journal of Physics 4 51 URL http://stacks.iop.org/1367-2630/ $4 / i=1 / a=351$

[12] Ido T et al. 2010 Plasma Physics and Controlled Fusion 52124025 URL http://stacks.iop. org/0741-3335/52/i=12/a=124025

[13] van Milligen B P et al. 2017 Nuclear Fusion 57056028 URL http://stacks.iop.org/ $0029-5515 / 57 / i=5 / a=056028$

[14] Sun P J et al. 2019 Physics of Plasmas 26 012304 (Preprint https://doi.org/10.1063/ 1.5049209) URL https://doi.org/10.1063/ 1.5049209

[15] Melnikov A V et al. 2011 Plasma and Fusion Research 6 2402030-2402030

[16] Hastie R J, Ramos J J and Porcelli F 2003 Physics of Plasmas 10 4405-4412 (Preprint https: //doi.org/10.1063/1.1612499) URL https: //doi.org/10.1063/1.1612499

[17] Dinklage A et al. 2018 Nature Physics 14 855-860

[18] Plunk G G, Connor J W and Helander P Journal of Plasma Physics $\mathbf{8 3} 715830404$

[19] Windisch T et al. 2017 Plasma Physics and Controlled Fusion 59105002 URL http://stacks. iop.org $/ 0741-3335 / 59 / i=10 / a=105002$

[20] Liu S C et al. 2018 Nuclear Fusion 58046002 URL http://stacks.iop.org/0029-5515/ $58 / i=4 / a=046002$

[21] Nührenberg C 2016 Nuclear Fusion 56076010 URL http://stacks.iop.org/0029-5515/ $56 / i=7 / a=076010$

[22] Liu S C, Liang Y, Drews P, Killer C, Knieps A, Xu G S, Wang H Q, Yan N, Han X, Höschen 
D, Krämer-Flecken A, Nicolai D, Satheeswaran G, Hammond K, Cai J Q, Charl A, Cosfeld J, Fuchert G, Gao Y, Geiger J, Grulke O, Henkel M, Hirsch M, Hoefel U, König R, Li Y, Neubauer O, Pasch E, Rahbarnia K, Rack M, Sandri N, Sereda S, Schweer B, Wang E H, Xu S and Gao X 2019 Nucl. Fusion 59 ISSN 17414326

[23] Liang Y et al. 2017 Nuclear Fusion 57066049 URL http://stacks.iop.org/0029-5515/ $57 / i=6 / a=066049$

[24] Krämer-Flecken A et al. 2011 Plasma Physics and Controlled Fusion $\mathbf{5 3} 074020$ URL http://stacks.iop.org/0741-3335/53/i=7/ $\mathrm{a}=074020$

[25] Krämer-Flecken A et al. 2017 Nuclear Fusion 57066023 URL http://stacks.iop.org/ $0029-5515 / 57 / i=6 / a=066023$

[26] Krämer-Flecken A, Han X, Windisch T, Cosfeld J, Drews P, Fuchert G, Geiger J, Grulke O, Killer C, Knieps A, Liang Y, Liu S and Rack M 2019 Plasma Phys. Control. Fusion 61054003 ISSN 0741-3335 URL http://stacks.iop.org/ $0741-3335 / 61 / i=5 / a=054003$ ? key=crossref . 9582d77a3c16b40788f05da641dc1204

[27] Drews P et al. 2017 Nuclear Fusion 57126020 URL http://stacks.iop.org/0029-5515/ $57 / i=12 / a=126020$

[28] Pasch E, Beurskens M N A, Bozhenkov S A, Fuchert G, Knauer J and Wolf R C 2016 Review of Scientific Instruments 87 11E729 (Preprint https://aip.scitation.org/doi/ pdf/10.1063/1.4962248) URL https://aip. scitation.org/doi/abs/10.1063/1.4962248

[29] Hirshman et al. 1986 The Physics of Fluids 29 2951-2959 (Preprint https://aip. scitation.org/doi/pdf/10.1063/1.865495) URL https://aip.scitation.org/doi/abs/ $10.1063 / 1.865495$

[30] Knauer J et al. 2016 A new dispersion interferometer for the stellarator wendelstein 7-x 43rd European Physical Society Conference on Plasma Physics (Leuven, Belgium) p 4.017

[31] Conway G D et al. 2008 Plasma Physics and Controlled Fusion 50124026 URL http://stacks. iop.org $/ 0741-3335 / 50 / i=12 / a=124026$

[32] Ch P Ritz C P et al. 1988 Review of Scientific Instruments 59 1739-1744 (Preprint https: //doi.org/10.1063/1.1140098) URL https: //doi.org/10.1063/1.1140098

[33] Prisiazhniuk D et al. 2017 Plasma Physics and Controlled Fusion 59025013 URL http://stacks.iop.org/0741-3335/59/i=2/ $a=025013$

[34] Scott B D 2005 Physics of Plasmas 12 062314 (Preprint https://doi.org/10.1063/ 1.1917866) URL https://doi.org/10.1063/ 1.1917866

[35] Zweben S J et al. 2015 Nuclear Fusion 55093035 URL http://stacks.iop.org/ $0029-5515 / 55 / i=9 / a=093035$

[36] Bozhenkov S A, Otte M, Biedermann C, Jakubowski M, Lazerson S A, Sunn Pedersen $\mathrm{T}$ and Wolf R C 2019 Nucl. Fusion 59 ISSN 17414326

[37] Gao Y, Jakubowski M W, Drewelow P, Pisano F, Puig Sitjes A, Niemann H, Ali A and Cannas B 2019 Nucl. Fusion 59 ISSN 17414326

[38] Burrell K H 1999 Phys. Plasmas 6 4418-4435 ISSN 1070664X

[39] Grenfell G, Van Milligen B P, Losada U, Ting W, Liu B, Silva C, Spolaore M and Hidalgo C 2019 Nucl. Fusion 59 ISSN 17414326 URL https: //doi.org/10.1088/1741-4326/aaf034 


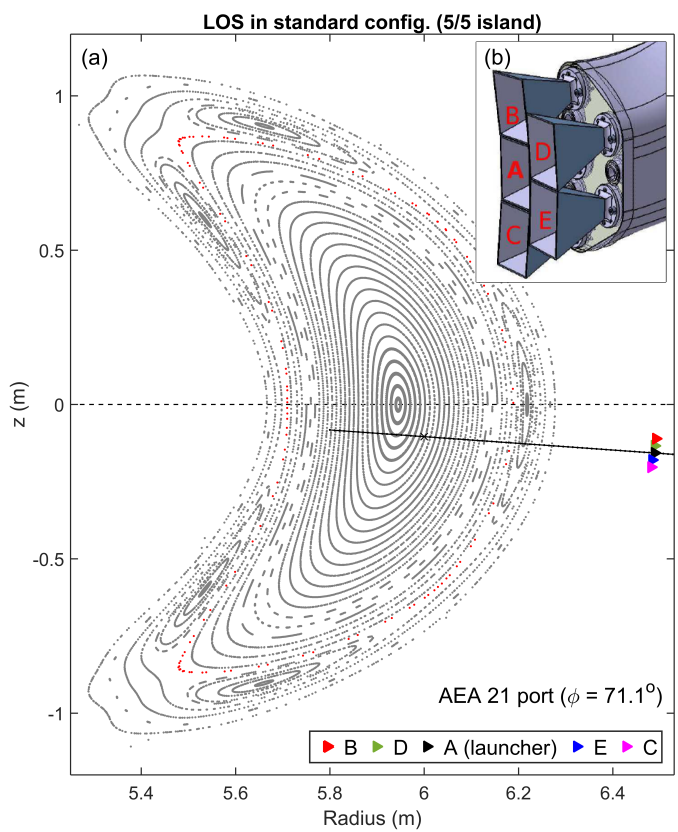

Figure 1. (a) Poincaré calculations at the cross-section of PCR's port (AEA21) in a standard configuration. The locations of PCR's launcher and receivers are marked as the colored triangles. The sight's line of launcher is labeled by the black line. (b) Zoomed view of the PCR's antenna array.
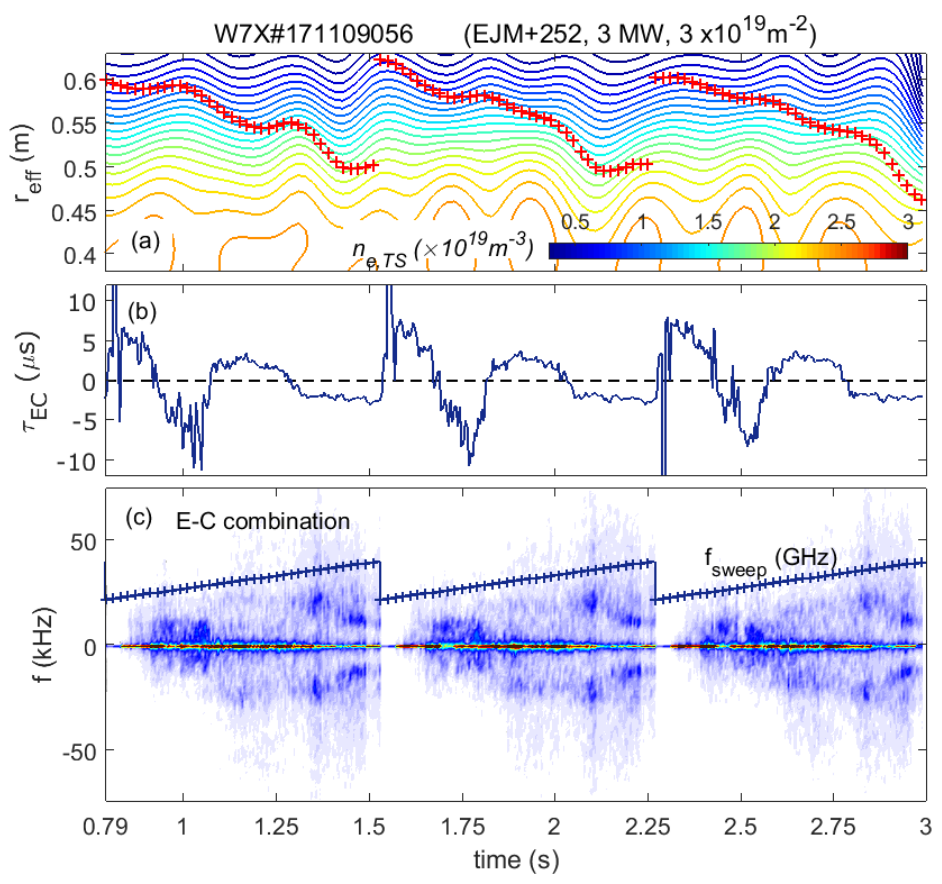

Figure 2. Time traces of (a) the contoured density profile with the PCR's cutoff position as marked by the red crosses, (b) the delay time evolution in the antenna E-C combination, and (c) the cross power spectrum of E-C combination. The incident frequency of PCR is indicated as the blue crosses. 


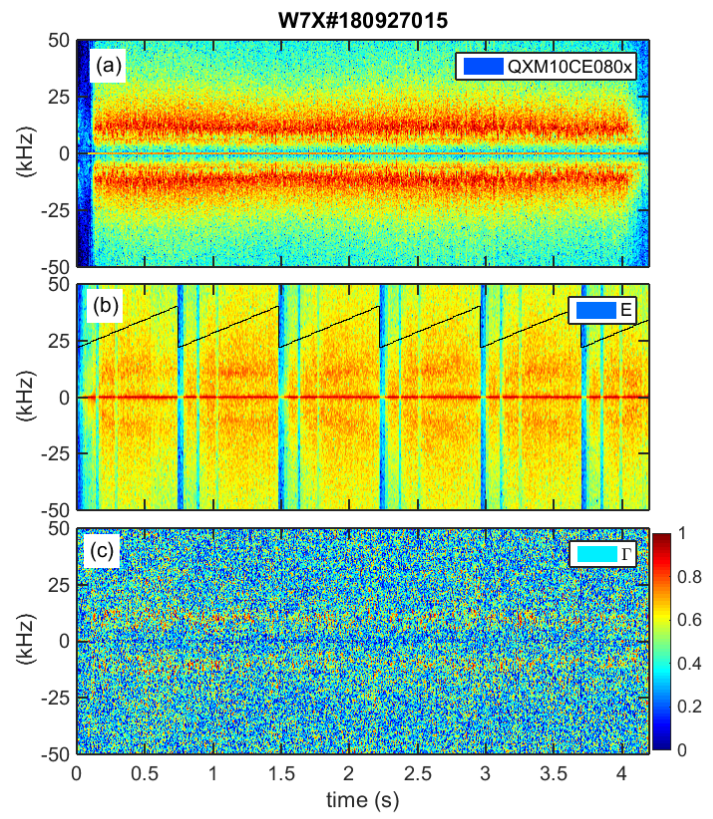

Figure 3. Auto-power spectra of (a) the magnetic fluctuations measured by the Mirnov coil, and (b) the autopower spectrum density in the PCR receiver-E. (c) The cross correlation coefficient between antenna E of PCR and Mirnov signal.
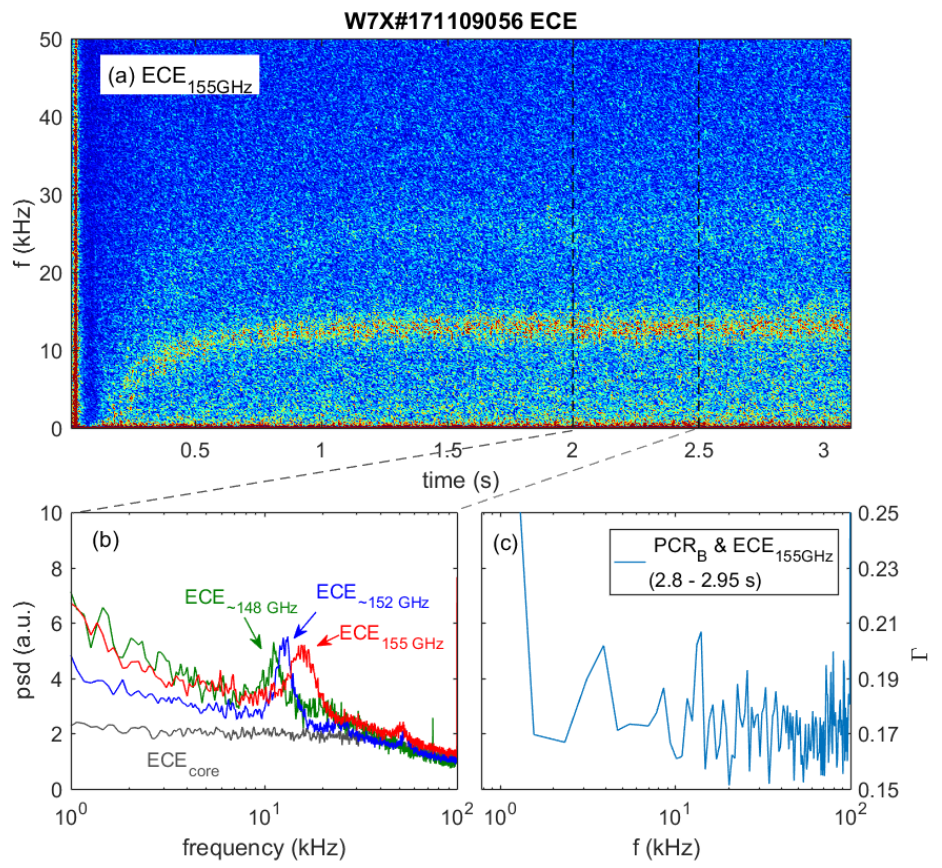

Figure 4. (a) The auto-power spectrum density of ECE edge channel, where the ECE frequency is $155 \mathrm{GHz}$. (b) The averaged power spectral density within the time window of $\mathrm{t}=2 \sim 2.5 \mathrm{~s}$. Both the core and edge ECE channels are overlapped with colored lines. (c) Cross-correlation coefficient between PCR receiver-B and ECE signal in $\mathrm{f}_{\mathrm{ECE}}=155 \mathrm{GHz}$. 


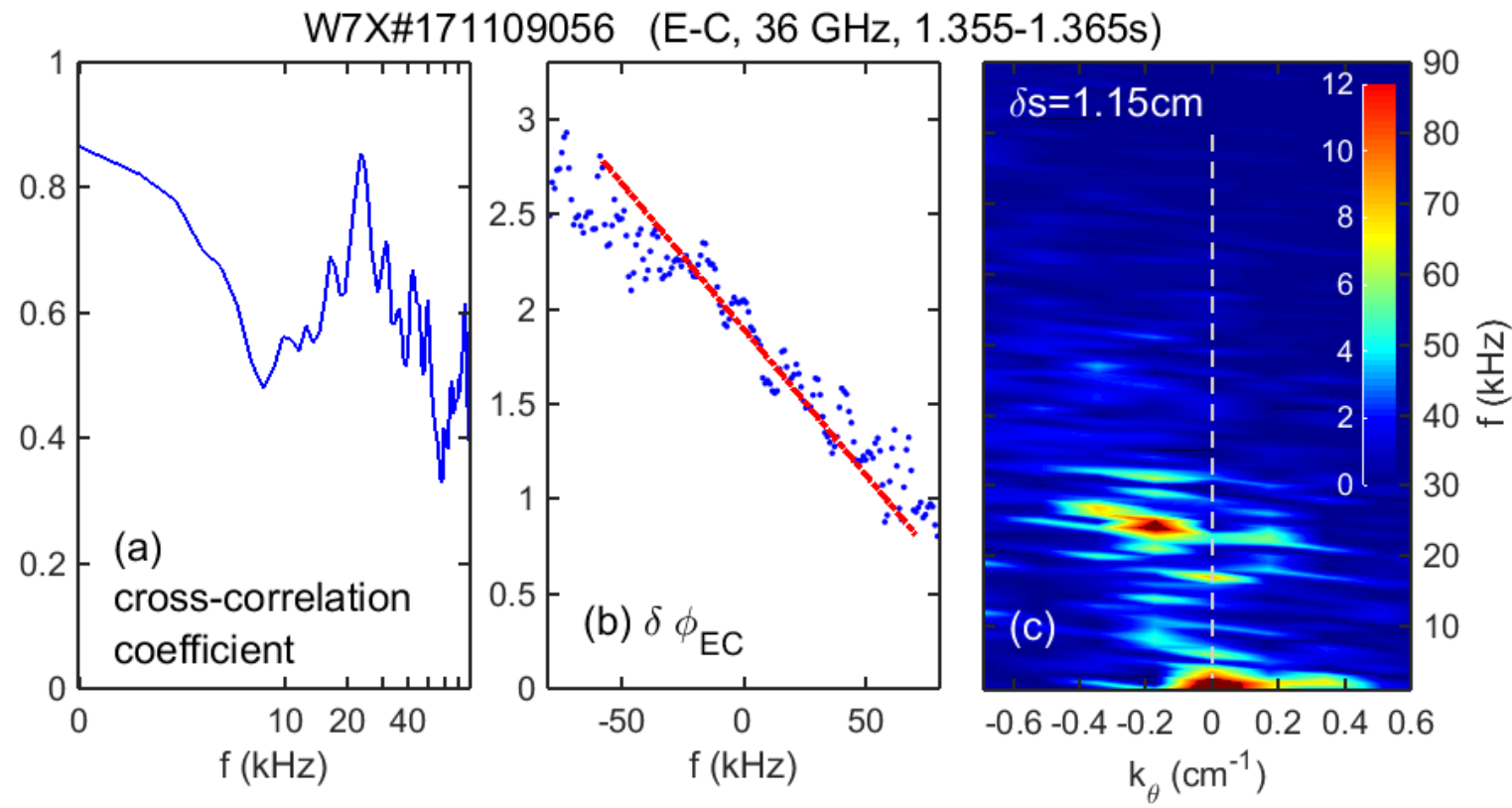

Figure 5. (a) The cross-correlation coefficient in PCR receiver E-C combination. The incident frequency in this time window is $\mathrm{f}=36 \mathrm{GHz}$. (b) The cross-phase profile at the same time window. (c) The frequencywavenumber spectral density $\left(\mathrm{S}_{\mathrm{k}, \mathrm{f}}\right)$. The distance at the corresponding cutoff layer is $\delta \mathrm{s}=1.15 \mathrm{~cm}$, and the negative wavenumber indicates an electron diamagnetic drift direction. 

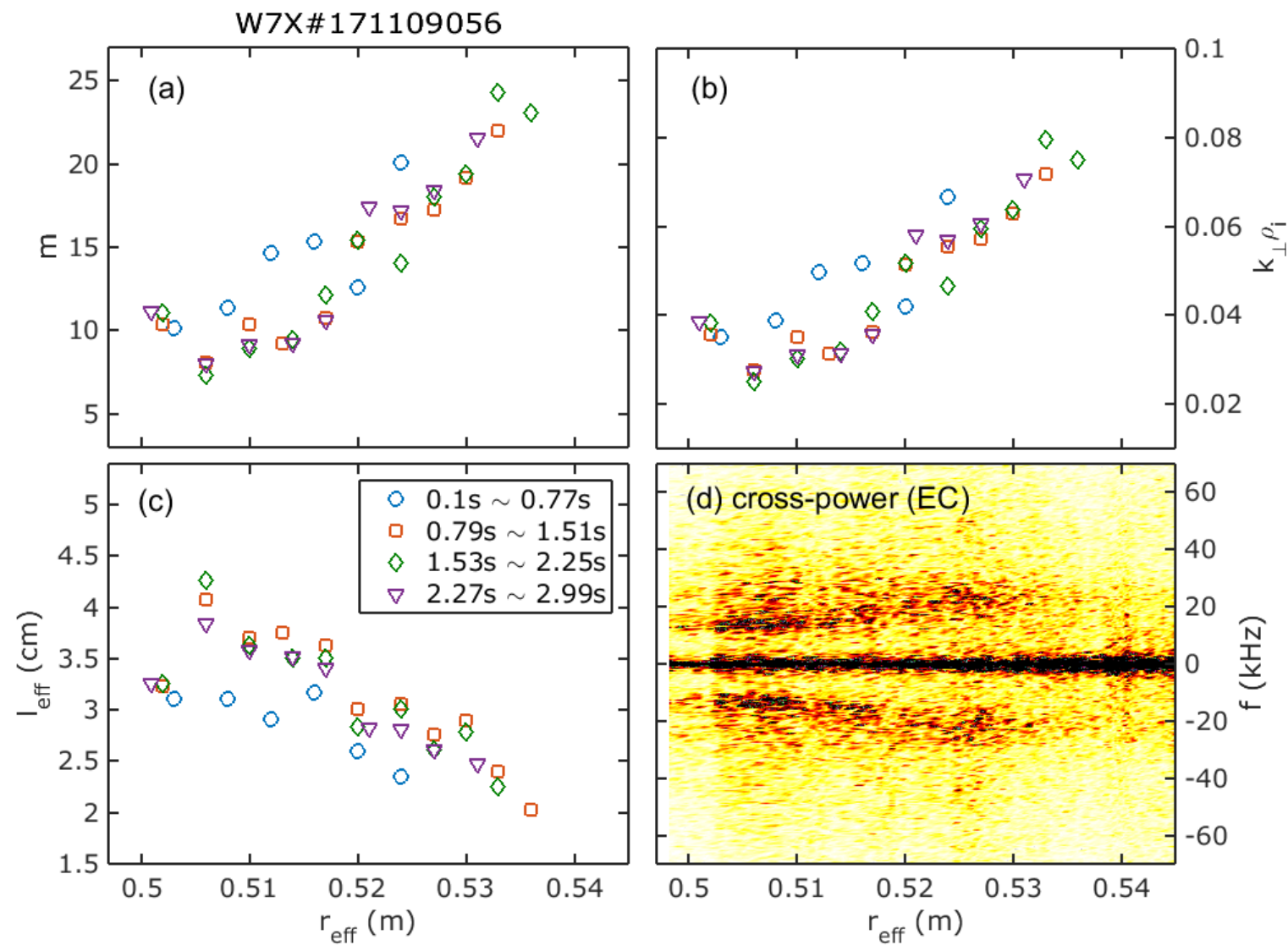

Figure 6. Calculations of (a) the poloidal mode number $(\mathrm{m})$, (b) the normalized gyroradius $\mathrm{k}_{\perp} \rho_{\mathrm{i}}$, (c) the effective correlation length $\mathrm{l}_{\mathrm{eff}}$, and (d) the radial distribution for the cross-power spectrum of E-C combination. 


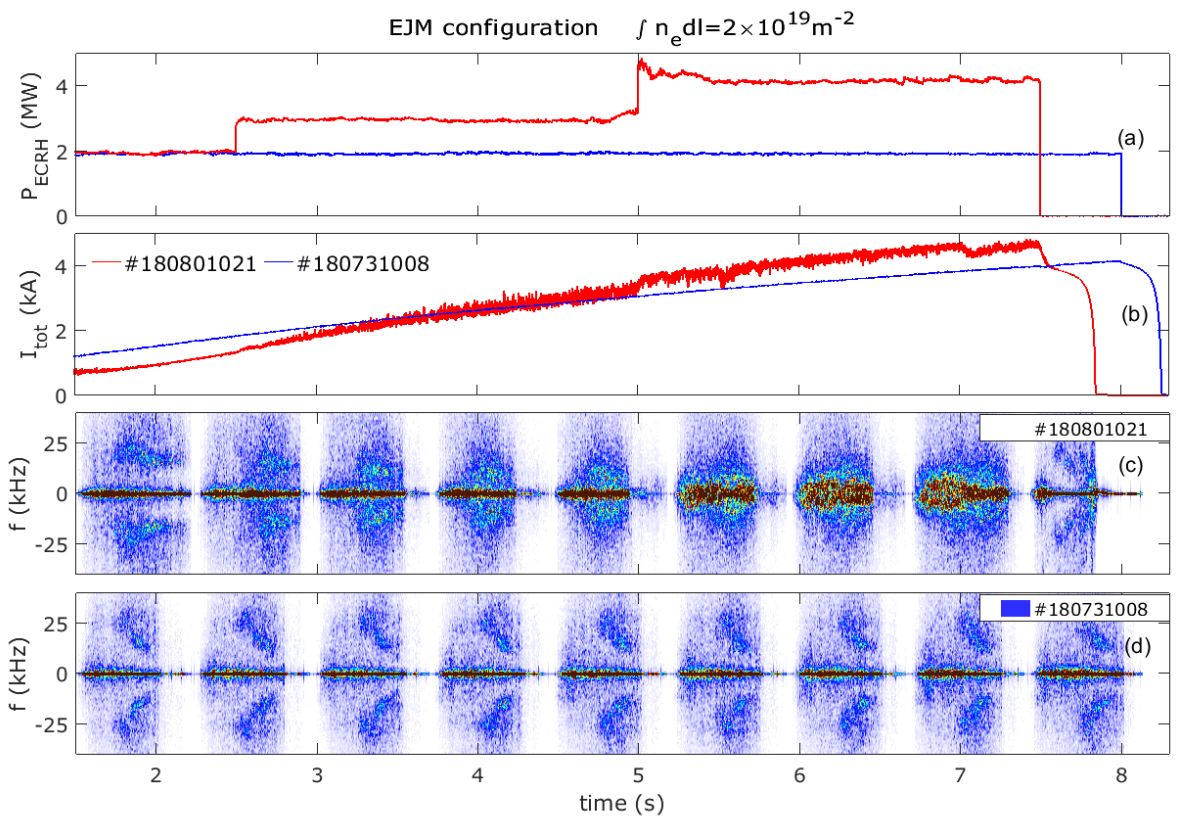

Figure 7. The time traces of (a) $\mathrm{P}_{\mathrm{ECRH}}$ and (b) $\mathrm{I}_{\text {tot }}$ in two discharges, which are indicated as the blue (\#180801021) and the red (\#180731008) color respectively. The cross-power spectrum using the receiver E-C combination in (c) \#180801021 and (d) \#180731008.

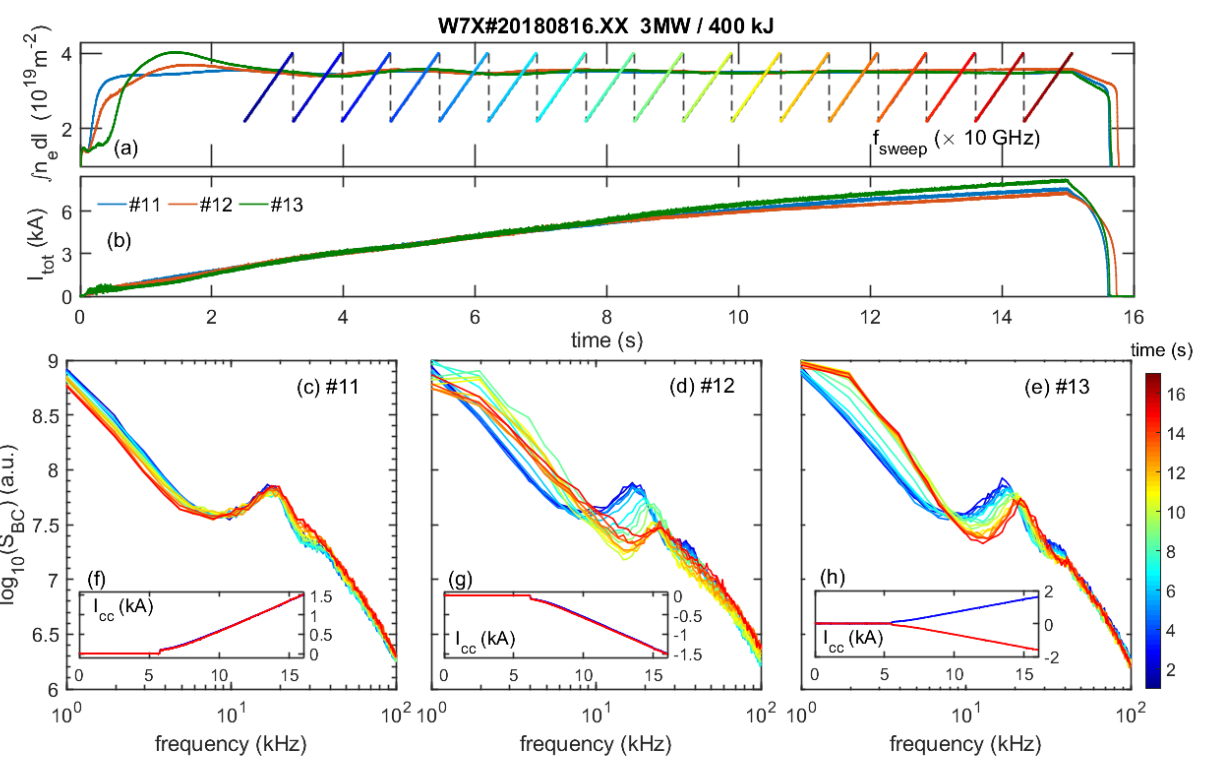

Figure 8. Time traces of (a) Line-integrated density overlapped with the PCR sweeping frequency. (b) and the total current. Figure (c, d, e) are the cross-power spectra of PCR in each sweeping cycle. The sub-figures (f) to (h) indicate the time trace of control coil currents $\left(\mathrm{I}_{\mathrm{cc}}\right)$ respectively. 


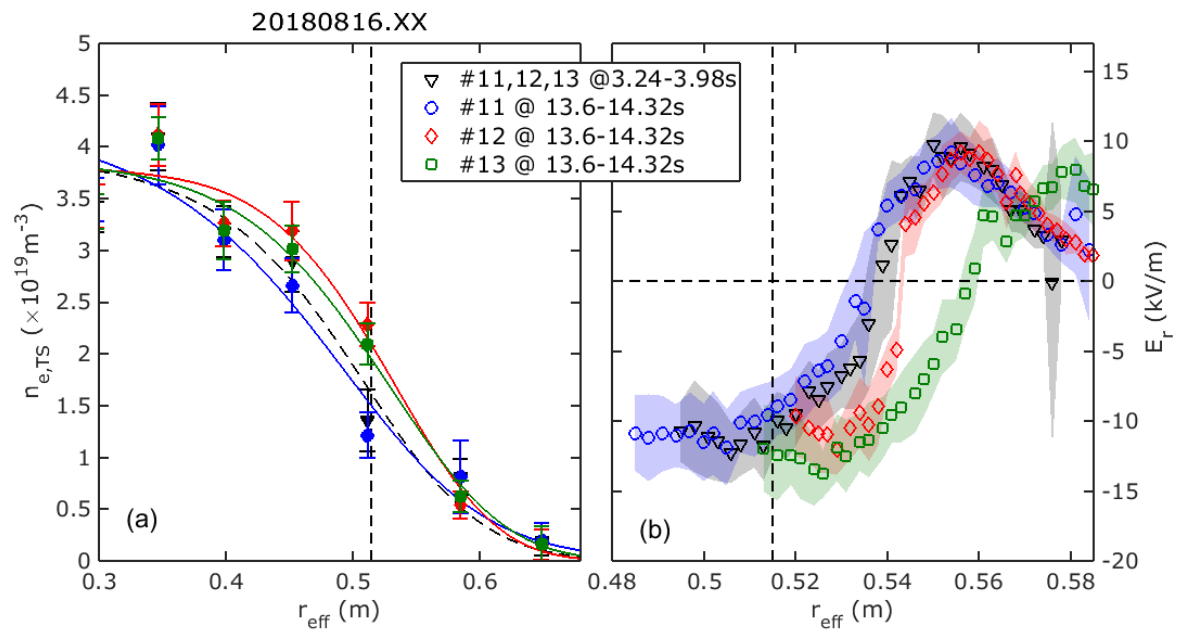

Figure 9. (a) The electron density profiles at different $I_{c c}$ scans, the profiles are measured via TS of data version 8. The vmec reference index is "vmec-ref-168" for a plasma $\beta=0 \%$ in the standard configuration. (b) The $E_{\mathrm{r}}$ profiles whose colors and symbols are related to the same time window of figure (a). The LCFS is indicated by the vertical black dash line individually. 